BMJ Open

Respiratory

Research

\title{
Microbial contamination of domiciliary nebulisers and clinical implications in chronic obstructive pulmonary disease
}

\author{
S Jarvis, ${ }^{1} \mathrm{P}$ W Ind, ${ }^{1} \mathrm{C}$ Thomas, ${ }^{2} \mathrm{~S}$ Goonesekera, ${ }^{2} \mathrm{R}$ Haffenden, ${ }^{3}$ A Abdolrasouli, ${ }^{2}$ \\ F Fiorentino, ${ }^{4} \mathrm{R}$ J Shiner ${ }^{1}$
}

To cite: Jarvis S, Ind PW, Thomas C, et al. Microbial contamination of domiciliary nebulisers and clinical implications in chronic obstructive pulmonary disease. BMJ Open Resp Res 2014;1:e00018.

doi:10.1136/bmjresp-2013000018

Received 18 November 2013 Revised 23 January 2014 Accepted 26 January 2014

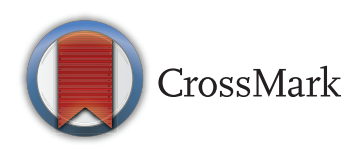

${ }^{1}$ Department of Respiratory Medicine, Imperial College Healthcare NHS Trust, Hammersmith Hospital, London, UK

2Department of Microbiology, Hammersmith Hospital, London, UK

${ }^{3}$ Central London Community Healthcare NHS Trust (CLCH), Charing Cross Hospital, London, UK ${ }^{4} \mathrm{NHLI}$, Department of Cardiothoracic Surgery, Hammersmith Hospital, Imperial College, London, UK

Correspondence to Dr R J Shiner;

r.shiner@imperial.ac.uk

\section{ABSTRACT}

Background and purpose: Domiciliary nebulisers are widely used in chronic obstructive pulmonary disease (COPD) but nebuliser cleaning practice has not been assessed in patients with COPD who are often elderly and may have severe disease and multiple comorbidities. We aimed to evaluate microbial contamination of home nebulisers used by patients with COPD.

Methods: Random microbiological assessment of domiciliary nebulisers was undertaken together with an enquiry into cleaning practices. We also examined the effectiveness of the trust-wide cleaning instructions in eradicating isolated microorganisms in a laboratory setting.

Results: The mean age of patients in this study was 71 (range 40-93) years, and in 68\% of patients a large number of significant comorbidities were present. Forty-four nebuliser sets were obtained and $73 \%$ were contaminated with microorganisms at $>100$ colony forming units/plate. Potentially pathogenic bacteria colonised 13 of the 44 nebulisers $(30 \%)$ and organisms isolated included Pseudomonas aeroginosa, Staphylococcus aureus, multidrug resistant Serratia marcesans, Escherichia coli and multiresistant Klebsiella spp, Enterobacteriaceae and fungus Fusarium oxysporum. Washing of nebuliser masks, chambers and mouthpieces achieved complete eradication of Gram-positive bacterial and fungal flora. Gramnegative organisms were incompletely eradicated, which may be attributed to the presence of biofilms. We also found that in patients with pathogenic organisms cultured on the nebuliser sets, there was a higher probability of occurrence of a COPD exacerbation with a mean number of exacerbations of $3.3(\mathrm{SD}=1)$ per year in the group in whom pathogens were isolated compared with $1.7(\mathrm{SD}=1.2)$

exacerbations per year in those whose sets grew nonpathogenic flora $(p=0.02)$.

Conclusions: Nebulisers contaminated with microorganisms are potential reservoirs delivering serious pathogens to the lung. Relationships between nebuliser contamination, clinical infection and exacerbations require further examination, but is a potential concern in elderly patients with COPD with comorbidities who fail to effectively maintain reasonable standards of nebuliser cleanliness.

\section{KEY MESSAGES}

Contaminated domiciliary nebuliser sets used by COPD patients pose a significant problem.

- COPD patients often have significant co-morbidities and other age-related factors which impair their ability to clean nebulisers adequately.

- Even recommended nebuliser washing techniques may not eradicate organisms which can form biofilms.

- This study draws attention to the fact that contaminated nebulisers are potential reservoirs and may be responsible for the delivery of serious pathogens to the lung.

\section{INTRODUCTION}

Nebulisers are used in acute exacerbations of chronic obstructive pulmonary disease (COPD) but many patients continue domiciliary nebulisers in the community. Chronic nebuliser use in management of COPD remains controversial since bronchodilation from $\beta-2$ agonists and anticholinergics by pressurised metered dose inhalers and dry powdered inhalers are considered comparable to nebulised therapy. ${ }^{1}$ However, many patients are not able to use hand-held inhaler devices effectively and in patients with multiple comorbidities and/or advancing age, nebulisers may represent a practical alternative. $^{2}$ In fact, many patients with COPD use domiciliary nebulisers, irrespective of whether they are obtained from their doctors or self-purchased. ${ }^{3}$ The British Thoracic Society advises replacement of nebuliser mouthpieces, masks and tubing at 3-6-month intervals and washing the nebuliser chamber at least daily. ${ }^{4}$ In addition, all patients should receive written instructions on nebuliser cleaning and how often this is necessary.

Contamination of domiciliary nebulisers with rates of $65 \%$ is reported in patients with cystic fibrosis and asthma where Gram-positive 
and Gram-negative microorganisms have been isolated..$^{5}$ One study demonstrated that in patients using domiciliary nebulisers, which included patients with COPD, one-third were contaminated with bacteria at concentrations which could be inhaled. ${ }^{7}$ Nebuliser contamination and the potential implications have not been well studied in the population with COPD, which is often elderly and faces challenges with comorbidities, mobility, eyesight and impaired manual dexterity, and which may impact on correct cleaning practices.

We evaluated microbial contamination of home nebulisers used by patients with COPD. Random microbiological assessment of nebulisers was undertaken together with enquiry into cleaning practices, as part of routine audit. We also examined the effectiveness of trust-wide cleaning instructions in eradicating isolated microorganisms in a laboratory setting.

\section{METHODS}

Patients with nebulisers were on our local COPD nebuliser database. All had received nebuliser cleaning information sheets instructing (1) daily nebuliser chamber washing in clean hot water, rinsing and air drying and (2) facemask or mouthpiece washing 2-3 times weekly in hot water, rinsing and air drying.

At a scheduled community respiratory nurse visit, patients' nebulisers, medication doses and regimens were reviewed. Patients were specifically questioned about the frequency and methods of nebuliser cleaning. Nebuliser chambers/tubing/masks/mouthpieces were collected (sometimes more than one set) and transported in sterile bags, directly to the microbiology laboratory. Clinical data collected included spirometry, use of long-term oxygen therapy (LTOT), details of exacerbations requiring antibiotics, comorbidities and social circumstances and care resources (which could potentially have affected cleaning). Exacerbation rates were not distributed normally. For comparisons, a Mann-Whitney two sample statistic test was used and because of the distribution of a number of exacerbations, we also dichotomised the number of exacerbations ( $\leq 1$ vs $>1)$ and used Fisher's exact test for a $2 \times 2$ frequency table.

\section{Culture methods}

Interior surfaces of nebuliser chambers, tubings, masks and mouthpieces were swabbed by rotating sterile watermoistened cotton tipped swabs for $10 \mathrm{~s}$. Swabs were inoculated onto chocolate, blood $\left(\begin{array}{lll}5-7 \% & \mathrm{CO}_{2}\end{array}\right)$ and MacConkey agar at $37^{\circ} \mathrm{C}$ and examined for colony growth at 24 and $48 \mathrm{~h}$. Nebuliser sets were washed (excluding tubing), as per Trust protocol, using hot water, air dried and reswabbed, cultured as before and read at 24 and $48 \mathrm{~h}$. Positive cultures were evaluated semiquantitatively: individual colonies were counted as colony forming units (CFU/plate) and categorised as: Scanty $( \pm)$ : $1-50$, Light $(+)$ : 50-100, Moderate $(++)$ : 100-200, and Heavy (+++): >200 CFU/plate.

\section{RESULTS}

Forty-four nebulisers were obtained from 37 patients (11 men), mean age 71 (range 40-93) years. Nebuliser models included PariBoy (PARI GmbH), Porta-neb (Respironics) and Medix Turboneb (Clement Clarke International) amongst others. Most patients had severe airflow obstruction by BTS COPD criteria. ${ }^{3}$ Mean (SD) forced expiratory volume in $1 \mathrm{~s}$ (available for 33 patients) was $0.9 \quad(0.4)$ L $\quad(39 \quad(15) \quad \%$ predicted $)$. Thirty-five per cent of patients required LTOT. Eighty-six per cent of nebulisers were used at least once daily and $66 \%$ of patients used their nebuliser twice or more daily. Significant comorbidities, present in 25 patients, included arthritis (42\%), impaired eyesight $(39 \%)$ and dementia (19\%). Twenty-nine patients required a carer at least once daily.

\section{Nebuliser contamination and cleaning efficacy}

Thirty-two of 44 collected nebuliser sets $(73 \%)$ were contaminated with microorganisms at $>100 \mathrm{CFU} /$ plate. Thirty-two masks/mouthpieces and 22 of the chambers were contaminated. Sixty per cent of microorganisms were normal skin and/or upper respiratory flora (including coagulase-negative Staphylococci, Bacillus spp, Diphtheroids and Candida spp). Potentially pathogenic bacteria colonised 13/44 (30\%) nebuliser sets and five patients had two sets of masks, tubings and chambers: similar types of organisms were isolated from both nebuliser sets (figure 1A). At least 100-200 CFU/plate were found on masks and mouthpieces. Heavy growth $(>200 \mathrm{CFU} /$ plate) was mainly found in nebuliser chambers (56\%). Potentially pathogenic organisms isolated included Pseudomonas aeruginosa, Staphylococcus aureus, lactose fermenting coliforms (LFC), including a multiresistant Serratia marcesans, Escherichia coli, multiresistant coliforms, Klebsiella, Enterobacteriaceae and, in one case, a potentially pathogenic fungus Fusarium oxysporum (table 1).

\section{Cleaning practices among patients and microorganisms isolated}

Eight-six per cent of patients used their nebuliser daily, $66 \%$ twice daily. Only 3/44 nebulisers were cleaned 'after each use', 7 'once daily' and 8 nebulisers had 'never' been cleaned. The remaining patients cleaned between alternate days and once weekly. The mean (SD) age of patients who 'never' cleaned their nebuliser was 76.1 (7.4) compared with 66.8 (12) years cleaning approximately once daily, but this was not statistically significant. Nebulisers from patients cleaning approximately once daily had fewer positive cultures (66\%) compared with those who had never cleaned their nebulisers $(86 \%)$, though this was not statistically significant.

In patients washing their nebulisers daily, normal flora and coagulase negative Staphylococcus were isolated and, in one patient, Pseudomonas aeruginosa. In 3/8 of nebuliser sets which had 'never been washed', E coli, Klebsiella, Enterobacteriaceae, Pseudomonas and $S$ aureus were isolated. Two nebulisers cleaned 'after each use' had a 
Figure 1 (A) Contamination of individual nebuliser components and contamination rates and organism types in patients with multiple nebulisers; five patients in the study had two sets of masks, tubings and chambers for their nebuliser, and a similar contamination rate in nebulisers from the same patient and the same types of organisms were isolated (+ denotes the presence of a microorganism, ++ moderate and +++heavy growth) (see

Culture methods section) [p] denotes a pathogenic organism. (B) Microbial contamination of nebuliser components prewashing and postwashing. (a)

\begin{tabular}{|c|c|c|c|c|c|c|}
\hline \multicolumn{1}{l|}{} & Mask (1) & Mask (2) & Chamber(1) & Chamber(2) & Tubing (1) & Tubing (2) \\
\hline Pt-1 & + & + & - & - & - & + \\
Pt-2 & + & ++ & + & - & - & - \\
Pt-3 & + & ++ & + & {$[\mathrm{p}]+++$} & - & ++ \\
Pt-4 & - & - & - & - & - & - \\
Pt-5 & ++ & {$[\mathrm{p}]+++$} & + & - & {$[\mathrm{p}]+++$} & ++ \\
\hline
\end{tabular}

(b)

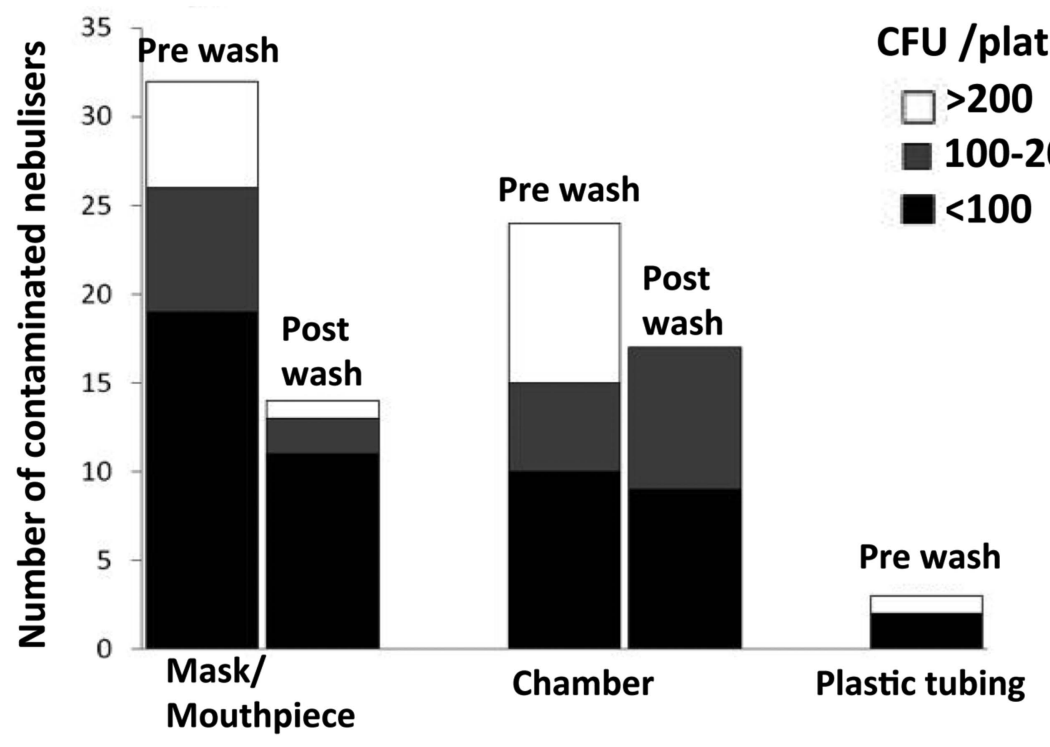

growth of coliforms ( $>100 \mathrm{CFU} /$ plate). No statistically significant difference was found in rates of contamination of nebulisers cleaned more or less frequently than advised.

Table 1 Pathogenic organisms isolated with the number of isolates in each component of the nebuliser set

\begin{tabular}{lllll}
\hline & Pathogenic organisms & Mask & Chamber & Tubing \\
\hline 1 & Enterobacteriaceae & +++ & +++ & - \\
2 & Fusarium oxysporum & - & Present & - \\
3 & Staphylococcus aureus & +++ & +++ & \\
4 & Enterobacteriaceae & - & +++ & - \\
5 & Pseudomonas & - & - & + \\
& aeruginosa & & & \\
6 & Enterobacteriaceae & - & +++ & - \\
7 & Escherichia coli & - & ++ & - \\
8 & Escherichia coli & - & ++ & - \\
9 & Serrratia marcesans & ++ & + & - \\
10 & Klebsiella pneumonia & - & ++ & - \\
11 & Enterobacteriaceae & - & +++ & - \\
\hline
\end{tabular}

Scanty $( \pm)$ : 1-50, Light (+): 50-100, Moderate (++): 100-200, and Heavy (+++): $>200$ CFU/plate.

CFU, colony forming units.

\section{Exacerbation rates}

Of the 44 nebuliser sets, 11 cultured potentially pathogenic organisms, 21 cultured non-pathogenic flora and 12 cultured no organisms. Information pertaining to the number of exacerbations requiring antibiotics per year was limited and was available for only a small number of patients overall (figure 2). The numbers of patients in each group with different numbers of exacerbations are shown in table 2 . The mean number of exacerbations was $3.3(\mathrm{SD}=1)$ in the pathogenic group $(\mathrm{n}=6$ patients in whom exacerbation data were available out of the 11 patients $), 1.7(\mathrm{SD}=1.2)$ in the non-pathogenic flora group $(\mathrm{n}=11$ patients $)$ and $1.6(\mathrm{SD}=1.3)$ in the group in which no microorganisms were isolated $(n=5)$. The median was 3,2 and 1 , respectively. We used a Mann-Whitney two sample statistic test due to the nature of the distribution of number of exacerbations. The Mann-Whitney test indicates that the two groups (pathogenic group vs non pathogenic flora) are statistically different $(p=0.02)$. Fisher's exact test gave $p=0.10$. This difference is as expected because Fisher's exact test uses less information (ie, has less power) than the Mann-Whitney test. This analysis suggested that patients with pathogenic organisms cultured on the nebuliser sets have a higher probability of a COPD 
Figure 2 Flow diagram depicting the number of nebuliser sets and records of exacerbation.

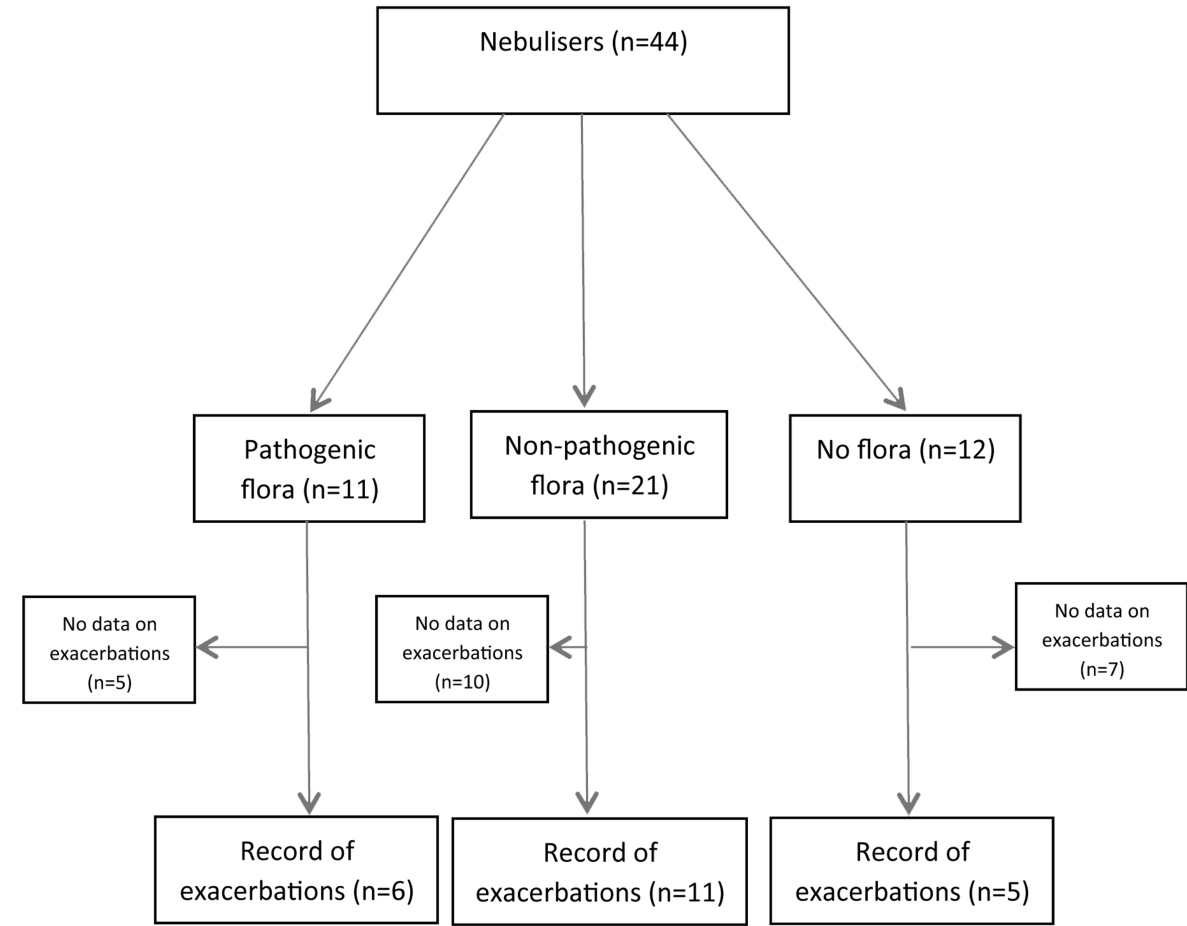

exacerbation and the effect appears to be large though not conclusive.

\section{Effectiveness of laboratory washing}

Washing achieved eradication of Gram-positive bacterial and fungal flora. Gram-negative organisms were incompletely eradicated (from $>200$ to $100-200 \mathrm{CFU} /$ plate postwashing (figure 1B). No new organisms were isolated after using tap water for washing, although plates for mycobacterial cultures were not set up.

\section{DISCUSSION}

This community audit of patients with COPD demonstrated a $73 \%$ frequency of domiciliary nebuliser contamination, which is comparable to one small study of patients with COPD using domiciliary nebulisers in which $81 \%$ of patients had equipment contamination with environmental organisms. ${ }^{8}$

Nebuliser chambers and masks were most frequently contaminated and $82 \%$ of patients did not clean their nebuliser as often as recommended, which may be

Table 2 Numbers of patients in each group with different numbers of exacerbations

\begin{tabular}{lllllll}
\hline & \multicolumn{7}{c}{ Number of exacerbations } \\
\cline { 2 - 7 } & Zero & One & Two & Three & Four & Five \\
\hline Pathogenic & 0 & 0 & 1 & 3 & 1 & 1 \\
Non-pathogenic & 2 & 3 & 2 & 4 & 0 & 0 \\
No organism & 1 & 2 & 0 & 2 & 0 & 0 \\
Total number & 3 & 5 & 3 & 9 & 1 & 1 \\
\hline
\end{tabular}

attributed to advancing age and comorbidities. Non-adherence to medications affects $40-45 \%$ of the elderly. ${ }^{9}$ Cognitive and visual decline, as well as reduced manual dexterity and mobility, can adversely affect selfmanagement skills. ${ }^{10}$ Here, $78 \%$ of our patients had carers and $37 \%$ required an organised care package. This raises the question of whether a carer feels able, or is allowed, to assist in nebuliser use and cleaning. Recently, published COPD guidance states that the ability of an individual and/or carer to use a nebuliser should be assessed before prescribing, and appropriate support and maintenance of equipment should be arranged. ${ }^{11}$

Optimal cleaning methods have not been addressed nationally since $1997 .{ }^{4}$ Nebuliser manufacturers recommend rinsing with soapy or warm water alone, with airdrying, after each use. Hypochlorite has been shown to improve cleaning efficacy and reduce contamination except in the case of Stenotrophomonas maltophilia. ${ }^{12}$ The possibility that its use can precipitate acute bronchoconstriction and compatibility of such chemicals with plastic nebuliser equipment remains a concern. ${ }^{13}$ Rosenfeld et $a l^{14}$ demonstrated in rigorous laboratory conditions that tap water cleaning, after every use, with air-drying eradicated organisms including $S$ aureus and Pseudomonas aeruginosa in $89 \%$ of cases, but not all studies have been able to show this. ${ }^{15}$ Other studies have shown no difference between cleaning reagents but improved cleaning efficacy with soaking the nebuliser no consensus on nebuliser cleaning, easier instructions are known to be associated with better compliance.

One study of patients aged between 1 and 88 years, who used domiciliary nebulisers, and included patients with components and then rinsing. ${ }^{14} 1617$ Although there is 
COPD, showed that Gram-positive bacteria, $P$ aeruginosa and $S$ marcesans were isolated. The concentrations found, if inhaled, could cause serious respiratory infections. ${ }^{7} \mathrm{We}$ isolated predominantly Gram-negative bacteria, which survive better in humid environments, and the growth of Pseudomonas and Enterobacter spp may relate to poor drying and strorage. ${ }^{9}$ The inability to completely eradicate Gram-negative bacteria after rigorous laboratory washing in this study and after use of detergents, acetic acid and quaternary ammonium compounds in another study remains a concern. ${ }^{15}$ Organisms resistant to washing, such as $P$ aeruginosa and $S$ aureus, can be transmitted to the airways and cause pneumonia. Effective equipment sterilisation has eradicated outbreaks of Gram-negative pneumonia. $^{18}$

Long-term pulmonary colonisation by the same clone of $P$ aeruginosa has been reported in patients with $\mathrm{COPD}^{19}$ while Klebsiella has been implicated in nosocomial outbreaks of severe pneumonia. ${ }^{20}$ We also isolated Fusarium oxysporum from one patient who had at least four COPD exacerbations per year. This organism can cause invasive infections in immunocompromised patients.

Although not the focus of this audit, the technicalities of nebulisation require attention. Temperature differences between commercially available nebulisers have not been systematically studied in the context of possible microbiological contamination. Contaminated nebulisers, generating 1-3 $\mu \mathrm{m}$ droplet diameters, could potentially deliver pathogenic organisms to terminal bronchioles. During nebulisation, machines such as PariBoy reduce their temperature from $24^{\circ} \mathrm{C}$ to $17^{\circ} \mathrm{C}$ after $8 \mathrm{~min}$ use altering the droplet size by $2.98 \mu \mathrm{m}$, potentially increasing lung pathogen deposition. The Multisonic nebuliser ( $\mathrm{GmbH} \& \mathrm{Co}$ ) increases temperature during nebulisation to $40^{\circ} \mathrm{C}$, but all bacteria isolated here could survive. Furthermore, surface plastic flaws may make nebuliser cleaning more difficult. Many pathogenic organisms identified in this audit, including $F$ oxysporum, ${ }^{21}$ can adhere to surfaces forming biofilms; complex aggregations of microorganisms. Gram-negative organisms such as Pseudomonas reside in multicellular clusters with individual cells in proximity within a biofilm, which is associated with antibacterial resistance, ${ }^{22}$ and it is possible that this may contribute to residual Gram-negative organisms seen despite washing.

Nebulisers contaminated with microorganisms are potential reservoirs delivering pathogens to the lung. Autoinfection is a potential concern in elderly patients with COPD with comorbidities who fail to effectively decontaminate their nebulisers. Relationships between nebuliser contamination, clinical infection and exacerbations require further examination. We found a statistically significant increase in the occurrence of exacerbations over 12 months in patients with potentially pathogenic organisms isolated from their nebulisers. The number of patients was small but the results were comparable to other studies. ${ }^{5}{ }^{7}$ The study suggests that patients with pathogenic organisms cultured on the nebuliser sets have a higher probability of occurrence of a COPD exacerbation. Although this study is not conclusive, the effect appears to be large and warrants further investigation in a larger prospective study with contemporaneous sputum collection.

This audit highlights medical and psychosocial issues in the population with COPD. Strategies to improve adherence to nebuliser cleaning as well as more effective, manageable techniques require rigorous evaluation.

Acknowledgements We would like to thank Dr Niall M Adams for his advice and the members of the CLCH community respiratory service.

Contributors SJ, PI and RS conceived the study and wrote the manuscript with PI. SG, SJ, CP and AA generated the results. RH helped with the data collection. FF has analysed the data and interpreted the results. All authors have read and approved the final manuscript.

Funding None.

Competing interests SJ is funded by the Wellcome Trust. FF is partly funded by the British Heart Foundation.

Ethics approval The local West London ethics committee were contacted and the project was approved as a routine audit and service evaluation.

Provenance and peer review Not commissioned; internally peer reviewed.

Data sharing statement No additional data are available.

Open Access This is an Open Access article distributed in accordance with the Creative Commons Attribution Non Commercial (CC BY-NC 3.0) license, which permits others to distribute, remix, adapt, build upon this work noncommercially, and license their derivative works on different terms, provided the original work is properly cited and the use is non-commercial. See: http:// creativecommons.org/licenses/by-nc/3.0/

\section{REFERENCES}

1. Brocklebank D, Ram F, Wright J, et al. Comparison of the effectiveness of inhaler devices in asthma and chronic obstructive airways disease: a systematic review of the literature. Health Technol Assess 2001;5:1-149.

2. Jarvis S, Ind PW, Shiner RJ. Inhaled therapy in elderly COPD patients; time for re-evaluation? Age Ageing 2007;36:213-18.

3. O'Driscoll BR, Kay EA, Taylor RJ, et al. Home nebulizers-can optimal therapy be predicted by laboratory studies. Respir Med 1990;84:471-7.

4. Wilson R, Muers MF. Running a domiciliary nebuliser service. Thorax 1997;52:S104-6.

5. Cohen HA, Kahan E, Cohen Z, et al. Microbial colonization of nebulizers used by asthmatic children. Pediatr Int 2006;48:454-8.

6. Pitchford KC, Corey M, Highsmith AK, et al. Pseudomonas species contamination of cystic fibrosis patients' home inhalation equipment. $J$ Pediatr 1987;111:212-16.

7. Barnes KL, Clifford R, Holgate ST, et al. Bacterial contamination of home nebuliser. BMJ (Clin Res Ed) 1987;295:812.

8. Higgs $C M B$, Jones $P$, Tanser AR. Bacterial contamination of home nebulisers. BMJ (Clin Res Ed) 1987;295:1281-2.

9. MacLaughlin EJ, Raehl CL, Treadway AK, et al. Assessing medication adherence in the elderly-which tools to use in clinical practice? Drugs Aging 2005;22:231-55.

10. Arlt S, Lindner R, Rösler A, et al. Adherence to medication in patients with dementia: predictors and strategies for improvement. Drugs Aging 2008;25:1033-47.

11. Chronic obstructive pulmonary disease (updated) 2013. http://www. nice.org.uk

12. Reychler G, Leonard A, Van Ossel C, et al. Impact of hypochlorite-based disinfection on bacterial contamination of cystic fibrosis patients' home-nebulisers. J Hosp Infect 2009;72:351-7.

13. Deschamps D, Soler P, Rosenberg N, et al. Persistent asthma after inhalation of a mixture of sodium hypochlorite and hydrochloric acid. Chest 1994;105:1895-6.

14. Rosenfeld M, Joy $P$, Nguyen $C D$, et al. Cleaning home nebulizers used by patients with cystic fibrosis: is rinsing with tap water enough? J Hosp Infect 2001;49:229-30. 
15. Chatburn RL, Kallstrom TJ, Bajaksouzian S. A comparison of acetic acid with a quaternary ammonium compound for disinfection of hand-held nebulizers. Respir Care 1988;33:179-87.

16. Blau $\mathrm{H}$, Mussaffi $\mathrm{H}$, Mei Zahav M, et al. Microbial contamination of nebulizers in the home treatment of cystic fibrosis. Child Care Health Dev 2007;33:491-5.

17. Tai $\mathrm{C}-\mathrm{H}$, Lin N-T, Peng T-C, et al. Cleaning small-volume nebulizers: the efficacy of different reagents and application methods. J Nurs Res 2011;19:61-7.

18. Pierce AK, Sanford JP, Thomas GD, et al. Long-term evaluation of decontamination of inhalation-therapy equipment and the occurrence of necrotizing pneumonia. New Engl J Med 1970;282:528-31.
19. Martínez-Solano L, Macia MD, Fajardo A, et al. Chronic Pseudomonas aeruginosa infection in chronic obstructive pulmonary disease. Clin Infect Dis 2008;47:1526-33.

20. Takigawa K, Fujita J, Negayama K, et al. Nosocomial outbreak of Pseudomonas cepacia respiratory infection in immunocompromised patients associated with contaminated nebulizer devices. Kansenshogaku Zasshi 1993;67:1115-25.

21. Retuerto MA, Szczotka-Flynn L, Ho D, et al. Efficacy of Care Solutions Against Contact Lens-Associated Fusarium Biofilms. Optom Vis Sci 2012;89:382-91.

22. Stewart PS, Costerton JW. Antibiotic resistance of bacteria in biofilms. Lancet 2001;358:135-8. 